\title{
MAPPING ONTO 2-DIMENSIONAL SPACES
}

\author{
BY DIX H. PETTEY \\ Communicated by Mary Ellen Rudin, July 1, 1971
}

In [3] the author showed that if $X$ is a connected, locally connected, locally compact topological space and $f$ is a 1-1 mapping of $X$ onto $E^{2}$, then $f$ is a homeomorphism. In this note, we present some significant extensions of the above result. An additional related result concerning 1-1 mappings is also given.

A topological space (not necessarily Hausdorff) will be said to be locally compact if each point has a compact neighborhood. By a mapping we will mean a continuous function. A mapping is said to be open if images of open sets are open. A mapping is compact if inverse images of compact sets are compact. If $K$ is a 2-cell, then $\mathrm{Bd} K$ and Int $K$ will denote, respectively, the boundary and interior of $K$, where $K$ is regarded as a 2-manifold with boundary.

THEOREM 1. Let $Y$ be a locally connected, locally compact metric space such that for each simple closed curve $J$ in $Y$ there is a 2-cell $K$ in $Y$ with $J=\mathrm{Bd} K$ and Int $K$ an open set in $Y$. If $X$ is a connected, locally connected, locally compact topological space and $f$ is a 1-1 mapping of $X$ onto $Y$, then $f$ is a homeomorphism.

The proof of this theorem depends upon a theorem of G. T. Whyburn [4, Theorem 7, p. 1430] and the above cited result of the author in [3]. Details will appear in another paper.

In [1] Edwin Duda defines a mapping $f$ to be reflexive compact if for each compact set $H$ in the domain space, $f^{-1}(f(H))$ is compact. In [2] Duda and Jack W. Smith define a mapping $f$ to be reflexive open if for each open set $V$ in the domain, $f^{-1}(f(V))$ is open. The following theorem generalizes results of Duda and Smith in [1] and [2].

THEOREM 2. Let $X$ and $Y$ be as in Theorem 1 . If $f$ is a reflexive compact (reflexive open) mapping of $X$ onto $Y$ then $f$ is compact (open).

The proof is obtained by using Theorem 1 and techniques from [1] and [2].

The following theorem establishes the existence of nontopological 1-1 mappings between certain kinds of spaces. The proof will appear elsewhere.

AMS 1970 subject classifications. Primary 54C10; Secondary 54F60, 57A05.

Key words and phrases. One-to-one mapping, open mapping, compact mapping, reflexive compact mapping, reflexive open mapping. 
THEOREM 3. Suppose that a metric space $Y$ is the union of $n$ 2-cells $K_{1}, K_{2}, \ldots, K_{n}$ such that for $1 \leqq i<j \leqq n, K_{i} \cap K_{j}=\operatorname{Bd} K_{i}=\operatorname{Bd} K_{j}$. If $n \geqq 3$, then there is a connected, locally connected, locally compact metric space which can be mapped onto Y by a nontopological 1-1 mapping.

It follows from Theorem 1 that if a metric space $Y$ is the union of two 2-spheres whose intersection is a point, then $Y$ is not the image, under a nontopological 1-1 mapping, of a connected, locally connected, locally compact topological space. In light of this, the following corollary to Theorem 3 is of interest.

COROLlARY. Suppose that a metric space $Y$ is the union of two 2-spheres having as their intersection a 2-cell. Then there is a connected, locally connected, locally compact metric space which can be mapped onto $Y$ by a nontopological 1-1 mapping.

\section{REFERENCES}

1. Edwin Duda, Reflexive compact mappings, Proc. Amer. Math. Soc. 17 (1966), 688-693. MR 33 \# 6589.

2. Edwin Duda and Jack W. Smith, Reflexive open mappings, Pacific J. Math. (to appear).

3. Dix H. Pettey, Mappings onto the plane, Trans. Amer. Math. Soc. 157 (1971), $297-309$.

4. G. T. Whyburn, On compactness of mappings, Proc. Nat. Acad. Sci. U.S.A. 52 (1964), 1426-1431. MR 31 \# 722.

Department of Mathematics, University of Missouri, Columbia, Missouri 65201 